\title{
Corrigendum
}

\section{Corrigendum to "Krasnosel'skii Type Hybrid Fixed Point Theorems and Their Applications to Fractional Integral Equations"}

\author{
H. M. Srivastava, ${ }^{1}$ Sachin V. Bedre, ${ }^{2,3}$ S. M. Khairnar, ${ }^{4}$ and B. S. Desale ${ }^{5}$ \\ ${ }^{1}$ Department of Mathematics and Statistics, University of Victoria, Victoria, BC, Canada V8W 3R4 \\ ${ }^{2}$ Department of Mathematics, Mahatma Gandhi Mahavidyalaya, Ahmedpur, District Latur, Maharashtra 413515, India \\ ${ }^{3}$ Research Scholar, Department of Mathematics, North Maharashtra University, Jalgaon, Maharashtra 415001, India \\ ${ }^{4}$ Department of Engineering Sciences, MIT Academy of Engineering, Alandi, Pune, Maharashtra 412105, India \\ ${ }^{5}$ Department of Mathematics, University of Mumbai, Mumbai, Maharashtra 400032, India
}

Correspondence should be addressed to Sachin V. Bedre; sachin.bedre@yahoo.com

Received 14 October 2014; Accepted 14 October 2014

Copyright (C) 2015 H. M. Srivastava et al. This is an open access article distributed under the Creative Commons Attribution License, which permits unrestricted use, distribution, and reproduction in any medium, provided the original work is properly cited.

In this note we correct some discrepancies that appeared in the paper by rewriting some statements and deleting proof of some theorems which already exist in our previous paper.

After examining different sections in the paper "Krasnosel'skii Type Hybrid Fixed Point Theorems and Their Applications to Fractional Integral Equations," we found some discrepancies. In this note, we slightly modify some of discrepancies by rewriting some statements and deleting proof of some theorems which already exist in our previous paper to achieve our claim.

We rewrite page 1, left side, line 1-line 4 (from Introduction), as follows.

The main result of Nieto and Rodríguez-López [1] is the following hybrid fixed point theorem.

We rewrite page 1, left side, line 1-line 2 (from bottom), as follows.

Another version of the above fixed point theorem can be stated as follows.

We rewrite page 2, left side, line 22-line 39, as follows.

The fixed point result of Heikkilä and Lakshmikantham [3] which originates all the above theoretical results differentiates in the convergence criteria of the sequence of iterations of the monotone mapping is as follows.

We rewrite page 2, left side, line 1-line 7 (from bottom), and right side, line 1-line 13, as follows.
Recently, Dhage $[4,5]$ and Bedre et al. [6] have obtained the Krasnosel'skii type fixed point theorems for monotone mappings.

We rewrite page 2, right side, line 11-line 12 (from bottom), as follows.

Now we consider the following definitions.

We rewrite page 3, left side, line 7-line 10 (from bottom), as follows.

We now state the basic hybrid fixed point results by Bedre et al. [6] using the argument from algebra, analysis, and geometry. The slight generalization of Theorem 4 and Dhage [8] using $M$-contraction is stated as follows.

We delete the proof of Theorem 14 and Corollary 15 and rewrite the statements as follows.

Theorem 14 (see Bedre et al. [6]). Let $(X, \preceq)$ be a partially ordered set and suppose that there exists a metric $d$ in $X$ such that $(X, d)$ is a complete metric space. Let $T: X \rightarrow X$ be a monotone function (nondecreasing or nonincreasing) such that there exists an $M$-function $\varphi_{T}$ such that

$$
d(T(x), T(y)) \leqq \varphi_{T}(d(x, y))
$$

for all comparable elements $x, y \in X$ satisfying $\varphi_{T}(r)<$ $r(r>0)$. Suppose that either $T$ is continuous or $X$ is such that if $x_{n} \rightarrow x$ is a sequence in $X$ whose consecutive terms 
are comparable, then there exists a subsequence $\left\{x_{n_{k}}\right\}_{k \in \mathbb{N}}$ of $\left\{x_{n}\right\}_{n \in \mathbb{N}}$ such that every term comparable to the limit $x$. If there exists $x_{0} \in X$ with $x_{0} \leqq T\left(x_{0}\right)$ or $x_{0} \geqq T\left(x_{0}\right)$, then $T$ has a fixed point which is unique if "every pair of elements in $X$ has a lower and an upper bound."

Corollary 15 (see Bedre et al. [6]). Let $(X, \preceq)$ be a partially ordered set and suppose that there exists a metric $d$ in $X$ such that $(X, d)$ is a complete metric space. Let $T: X \rightarrow X$ be a monotone function (nondecreasing or nonincreasing) such that there exists an $M$-function $\varphi_{T}$ and a positive integer $p$ such that

$$
d\left(T^{p}(x), T^{p}(y)\right) \leqq \varphi_{T}(d(x, y))
$$

for all comparable elements $x, y \in X$ satisfying $\varphi_{T}(r)<$ $r(r>0)$. Suppose that either $T$ is continuous or $X$ is such that if $x_{n} \rightarrow x$ is a sequence in $X$ whose consecutive terms are comparable, then there exists a subsequence $\left\{x_{n_{k}}\right\}_{k \in \mathbb{N}}$ of $\left\{x_{n}\right\}_{n \in \mathbb{N}}$ such that every term comparable to the limit $x$. If there exists $x_{0} \in X$ with $x_{0} \leqq T\left(x_{0}\right)$ or $x_{0} \geqq T\left(x_{0}\right)$, then $T$ has a fixed point which is unique if "every pair of elements in X has a lower and an upper bound."

We rewrite page 4, left side, line 7-line 15 (from bottom), as follows.

We now consider the following definition.

We rewrite page 4, right side, line 6-line 11 (from bottom), as follows.

The following Krasnosel'skii type fixed point theorem is proved in Dhage [5]. 


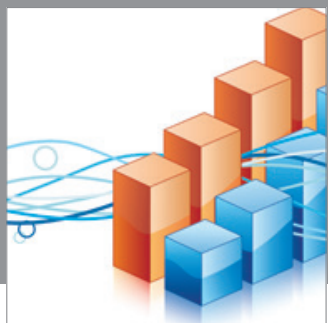

Advances in

Operations Research

mansans

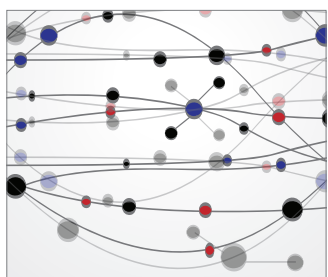

The Scientific World Journal
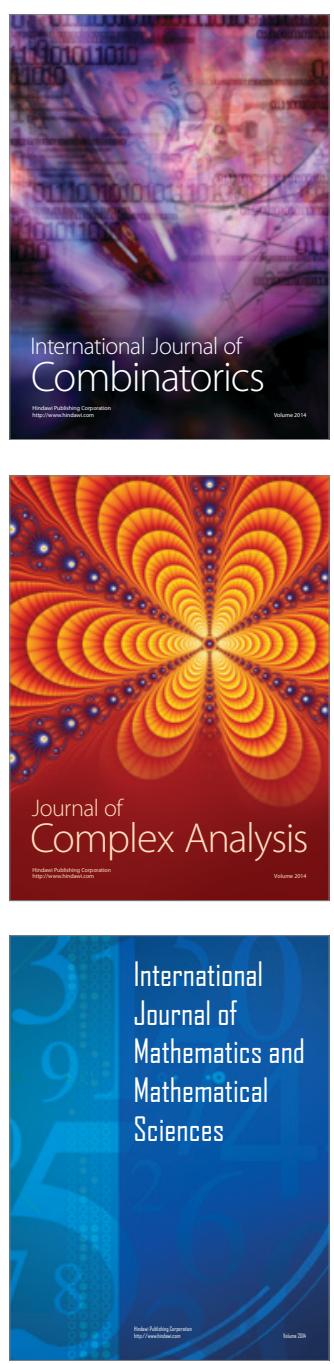
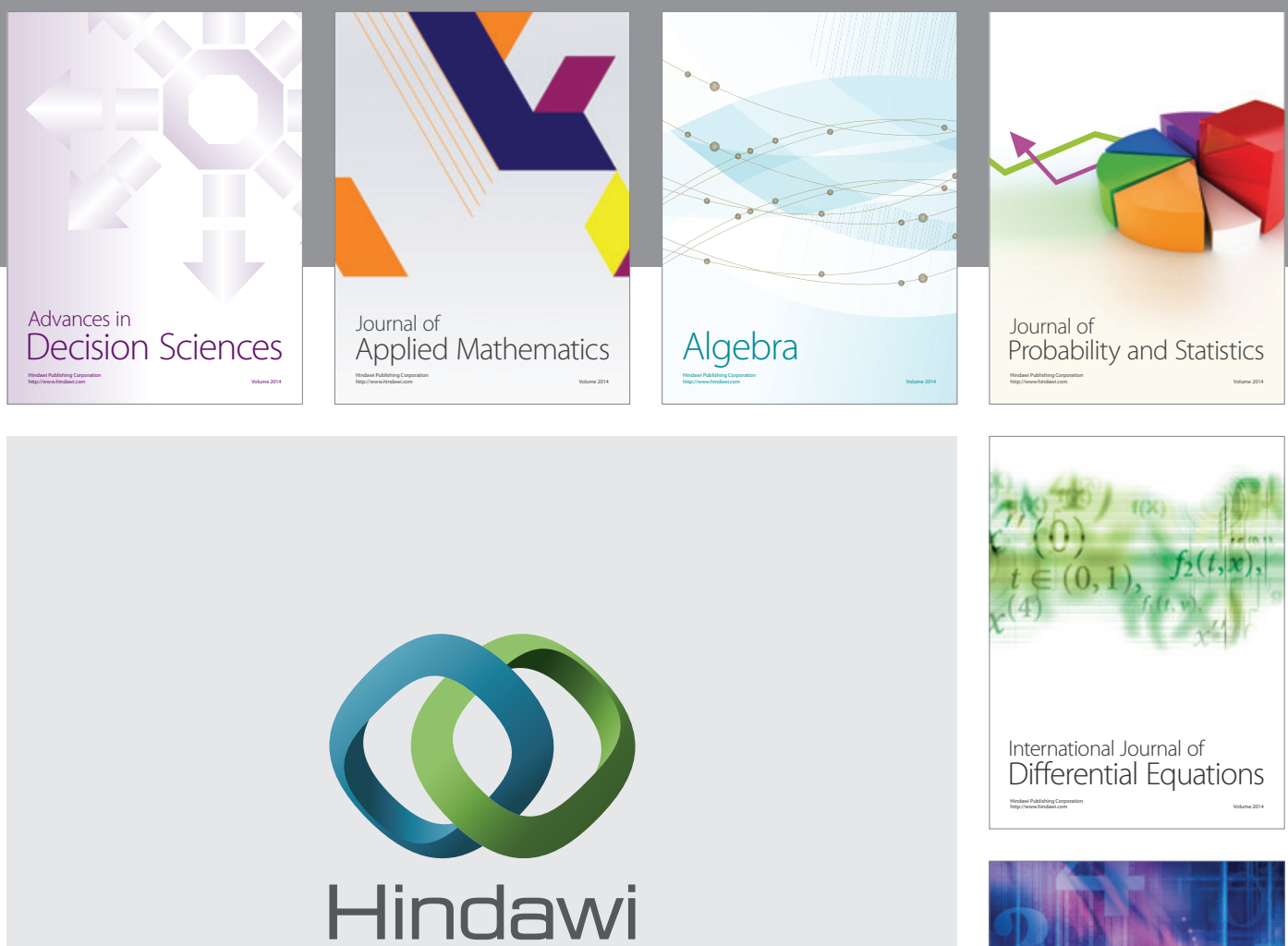

Submit your manuscripts at http://www.hindawi.com
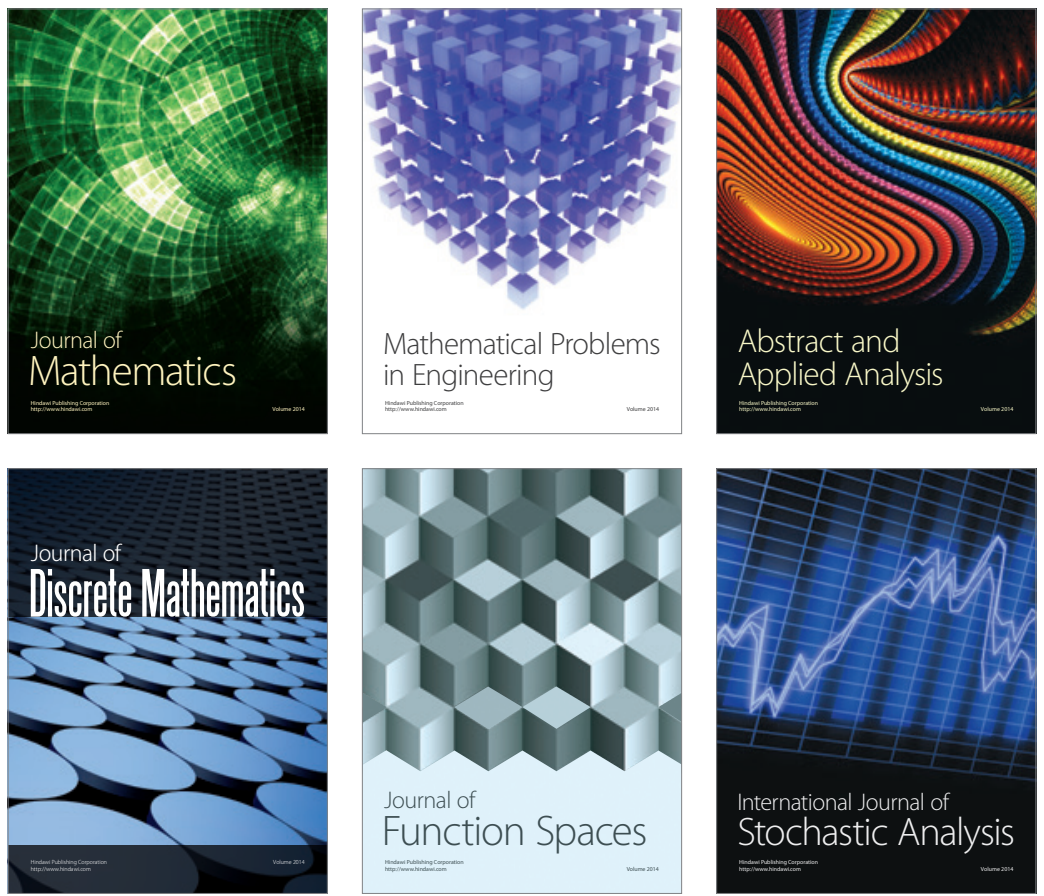

Journal of

Function Spaces

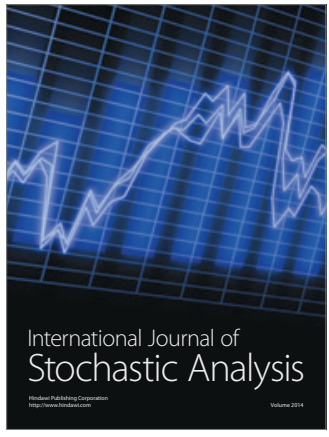

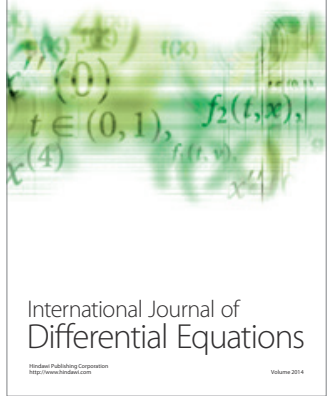
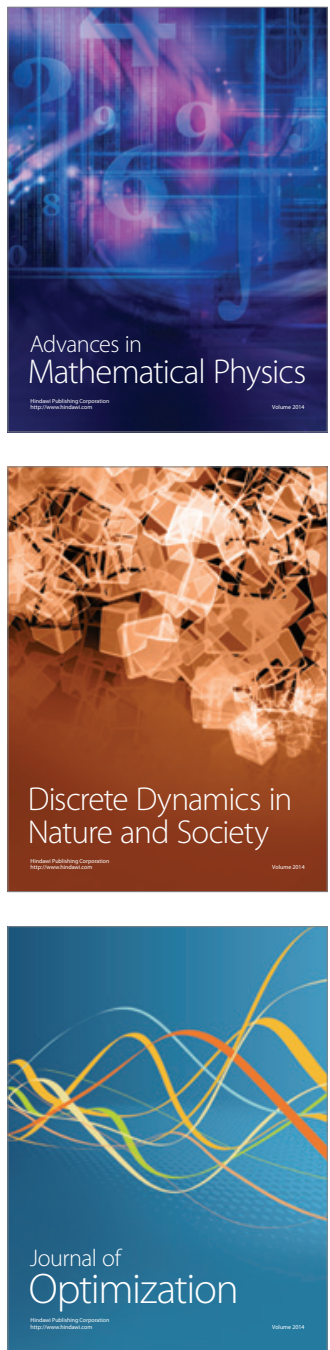